\title{
P01.10. Effects of caloric restriction combined with traditional chinese phytomedicine on the glucolipid metabolism in Wistar Rats with insulin resistance
}

\author{
$C L^{i *}, S L i^{2}, A$ Michalsen ${ }^{1}, J$ Qin $^{2}$ \\ From International Research Congress on Integrative Medicine and Health 2012 \\ Portland, Oregon, USA. 15-18 May 2012
}

\section{Purpose}

Insulin resistance (IR) and hyperlipemia are two important pathophysiological manifestations in metabolic diseases. This research aims to investigate the influence of complete caloric restriction (CR) combined with Chinese medicine decoction (ling-gui-zhu-gan,LGZG) on glucose and lipid metabolism in rats with IR.

\section{Methods}

48 male Wistar rats were randomly assigned by $3: 1$ ratio to a high-fat diet group, which was fed with a high-fat diet for 12 weeks, and a control group which received a standard diet, respectively. Rats in the high-fat diet group were randomized by a ratio of $1: 1: 1$ into a $C R$ group, a CR+LGZG group that additionally received a LGZG decoction, and a group which continued the high-fat diet. Rats in the CR+LGZG group were administered intragastrically LGZG decoction daily, and the other two groups received $3 \mathrm{ml}$ of saline. Outcomes were assessed at baseline, at the end of the $12^{\text {th }}$ week, and after the 3-day CR.

\section{Results}

At the end of the $12^{\text {th }}$ week, the rats with insulin resistance were established successfully in the high-fat diet group. After CR, fasting plasma glucose (FPG), fasting insulin (FINS) and insulin resistance index (IRI) were reduced by $10.0 \%(\mathrm{p}>0.05), 61.6 \%(\mathrm{p}<0.01)$ and $65.5 \%$ $(\mathrm{p}<0.01)$ compared with standard diet, while CR+LGZG led to reductions of $43.9 \%(\mathrm{p}<0.01), 73.4 \%(\mathrm{p}<0.01)$ and
85.9\% ( $\mathrm{p}<0.01)$. Compared with CR only, CR+LGZG led to significant decreases in FPG $(\mathrm{p}<0.01)$, FINS $(\mathrm{p}<0.05)$ and IRI $(\mathrm{p}<0.01)$. Blood lipids (CHO, TG, HDL-C, LDL$\mathrm{C})$ were significantly reduced in both $\mathrm{CR}$ only and $\mathrm{CR}$ +LGZG compared with standard diet $(\mathrm{p}<0.01)$, while there was no difference between CR with or without LGZG.

\section{Conclusion}

CR+LGZG decoction has greater effects on glucose metabolism than only CR. Both CR and CR + LGZG improve lipids to the same extent. The beneficial effect of an additional herbal medication during CR might be related to fasting-induced gluconeogenesis. Human studies on the beneficial impact of $\mathrm{CR}+$ Chinese medicine on diabetic patients are warranted.

\section{Author details}

${ }^{1}$ Charité, University Medical Centre Berlin, Berlin, Germany. ${ }^{2}$ The First Affiliated Hospital of Sun Yat-Sen University, Guangzhou, China.

Published: 12 June 2012

doi:10.1186/1472-6882-12-S1-P10

Cite this article as: Li et al:: P01.10. Effects of caloric restriction combined with traditional chinese phytomedicine on the glucolipid metabolism in Wistar Rats with insulin resistance. BMC Complementary and Alternative Medicine 2012 12(Suppl 1):P10. 